\title{
Self-training of dynamic touch: Striking improves judgment by wielding
}

\author{
Damian G. Stephen ANd Ryan ArZamarski \\ University of Connecticut, Storrs, Connecticut
}

\begin{abstract}
In traditional theories of perceptual learning, sensory modalities support one another. A good example comes from research on dynamic touch, the wielding of an unseen object to perceive its properties. Wielding provides the haptic system with mechanical information related to the length of the object. Visual feedback can improve the accuracy of subsequent length judgments; visual perception supports haptic perception. Such cross-modal support is not the only route to perceptual learning. We present a dynamic touch task in which we replaced visual feedback with the instruction to strike the unseen object against an unseen surface following length judgment. This additional mechanical information improved subsequent length judgments. We propose a self-organizing perspective in which a single modality trains itself.
\end{abstract}

Traditional theories of perceptual learning respect a division of labor among the sensory modalities. These modalities (e.g., vision, touch) are sensitive to changes in distinct sensory fields (e.g., light, pressure). In such theoretical accounts, each modality can pick up only a limited range of information, past the bounds of which another modality is needed for specification (Bahrick \& Lickliter, 2002; Lickliter, Bahrick, \& Markham, 2006). Independently, two modalities can glean only so much information; in concert, nonoverlapping information from the second modality requires integration with information from the first modality. That is, training of one modality requires the support of another modality. Despite intuitive appeal, this traditional formalism for perceptual learning neglects the flexibility available in the perceptual system (e.g., Van Orden, Holden, \& Turvey, 2003). Theories of perceptual learning have sought to prop one modality up with another but have overlooked the possibility that one modality may train itself.

Perceptual learning research in dynamic touch has investigated the role of visual information in haptic perception (e.g., Michaels, Arzamarski, Isenhower, \& Jacobs, 2008; Wagman, Shockley, Riley, \& Turvey, 2001; Withagen \& Michaels, 2005). In dynamic touch, participants wield an unseen object and judge its properties (e.g., length). When given visual feedback on their judgments following wielding, participants have been shown to improve subsequent judgments. That is, visual perception modulates haptic perception (see also Streit, Shockley, Morris, \& Riley, 2007). In the absence of visual information, haptic perception by wielding is thought to remain a constant function of the logarithms of the object's principal inertial moments (e.g., Fitzpatrick, Carello, \& Turvey, 1994). In this article, we present evidence that dynamic touch may have the capacity for generating its own new information above and beyond wielding. A perceptual system may train itself, rather than rely on other perceptual systems or even on extrinsic feedback from an experimenter (cf. Jacobs \& Michaels, 2002, 2007).

Self-training might proceed from the ability of one modality to sample from multiple pools of information. Multiple modalities may not be necessary to achieve perceptual learning if only there are multiple sources of information (Stoffregen \& Bardy, 2001). Typical dynamic touch experiments give the haptic system only one source of mechanical information-namely, wielding the object about the wrist. In the present study, we will give the haptic system two sources of mechanical informationnamely, wielding and striking. The role of mechanical contact from striking has been studied in dynamic touch, but only as a single exploratory style. The evidence from studies on both wielding and striking suggest that they provide different kinds of mechanical information about length (Barac-Cikoja \& Turvey, 1991; Carello, Fitzpatrick, \& Turvey, 1992; Chan \& Turvey, 1991). Research in dynamic touch has yet to evaluate the role of striking in modulating judgments by wielding. We anticipate that the provision of wielding and striking together will improve performance in length perception - that is, reduce discrepancies between perceived length $(P L)$ and actual length $(L)$. 


\section{METHOD}

\section{Participants}

Seventeen students ( 10 females, 7 males; mean age $=19.4$ years, $S D=0.3$ ) at the University of Connecticut participated in order to fulfill a course requirement. Fifteen were right-handed, and two were left-handed. Experimenters assigned 9 to the experimental condition and 8 to the control condition.

\section{Task, Apparatus, and Design}

The stimulus set comprised 20 wooden dowels, $1.3 \mathrm{~cm}$ in diameter (see Table 1). A black marker was used to mark the end of the first $9 \mathrm{~cm}$ of each dowel.

Figure 1A illustrates the experimental apparatus. A chair was arranged with an armrest on the right side. A curtain hung flush with the right shoulder of a seated participant. The participant's right hand extended beyond the curtain through a $20-\mathrm{cm}$ slit. A pulley system was placed along the length of a table positioned before the seated participant. The pulley system controlled the movement of a response marker in an anterior-posterior direction. The response marker could thus be positioned anywhere along the pulley system, from the edge of the table closest to the seated participant to $150 \mathrm{~cm}$ away from the seated participant.

One dowel was presented per trial. On each trial, the experimenter placed the first $9 \mathrm{~cm}$ of the dowel in the participant's right hand. To do so, the experimenter held the dowel just above the 9-cm mark on the dowel, controlling for any mechanical contact incidental to placement of the dowel. The participant was instructed to wield the dowel freely about the wrist and to judge its length. The participant
Table 1

Details of Stimuli

\begin{tabular}{crrrrr}
\hline Rod No. & $\begin{array}{c}\text { Length } \\
(\mathrm{cm})\end{array}$ & $\begin{array}{r}\text { Density } \\
\left(\mathrm{g} / \mathrm{cm}^{3}\right)\end{array}$ & Mass $(\mathrm{g})$ & $I_{1}\left(\mathrm{~g} * \mathrm{~cm}^{2}\right)$ & $I_{3}\left(\mathrm{~g} * \mathrm{~cm}^{2}\right)$ \\
\hline 1 & 20 & 0.30 & 8.00 & 1,067 & 267 \\
2 & 25 & 0.50 & 16.74 & 3,487 & 872 \\
3 & 30 & 0.74 & 29.27 & 8,781 & 2,196 \\
4 & 35 & 0.76 & 35.42 & 14,463 & 3,617 \\
5 & 40 & 0.35 & 18.82 & 10,037 & 2,510 \\
6 & 45 & 0.55 & 32.62 & 22,018 & 5,506 \\
7 & 50 & 0.57 & 37.96 & 31,633 & 7,909 \\
8 & 55 & 0.72 & 52.51 & 52,947 & 13,238 \\
9 & 60 & 0.53 & 42.42 & 50,903 & 12,727 \\
10 & 65 & 0.54 & 46.77 & 65,867 & 16,468 \\
11 & 70 & 0.53 & 49.60 & 81,013 & 20,254 \\
12 & 75 & 0.39 & 38.59 & 72,356 & 18,090 \\
13 & 80 & 0.71 & 75.75 & 161,598 & 40,401 \\
14 & 85 & 0.70 & 78.58 & 189,245 & 47,313 \\
15 & 90 & 0.49 & 58.92 & 159,082 & 39,771 \\
16 & 95 & 0.62 & 78.20 & 235,249 & 58,813 \\
17 & 100 & 0.62 & 82.07 & 273,564 & 68,392 \\
18 & 105 & 0.68 & 94.74 & 348,166 & 87,042 \\
19 & 110 & 0.70 & 101.97 & 411,275 & 102,819 \\
20 & 115 & 0.46 & 70.70 & 311,666 & 77,917 \\
\hline
\end{tabular}

was then instructed to indicate his or her judgment by moving the marker along the pulley system, so that the distance from the table's edge to the marker matched the length of the dowel. The experimenter recorded the length judgment.

A
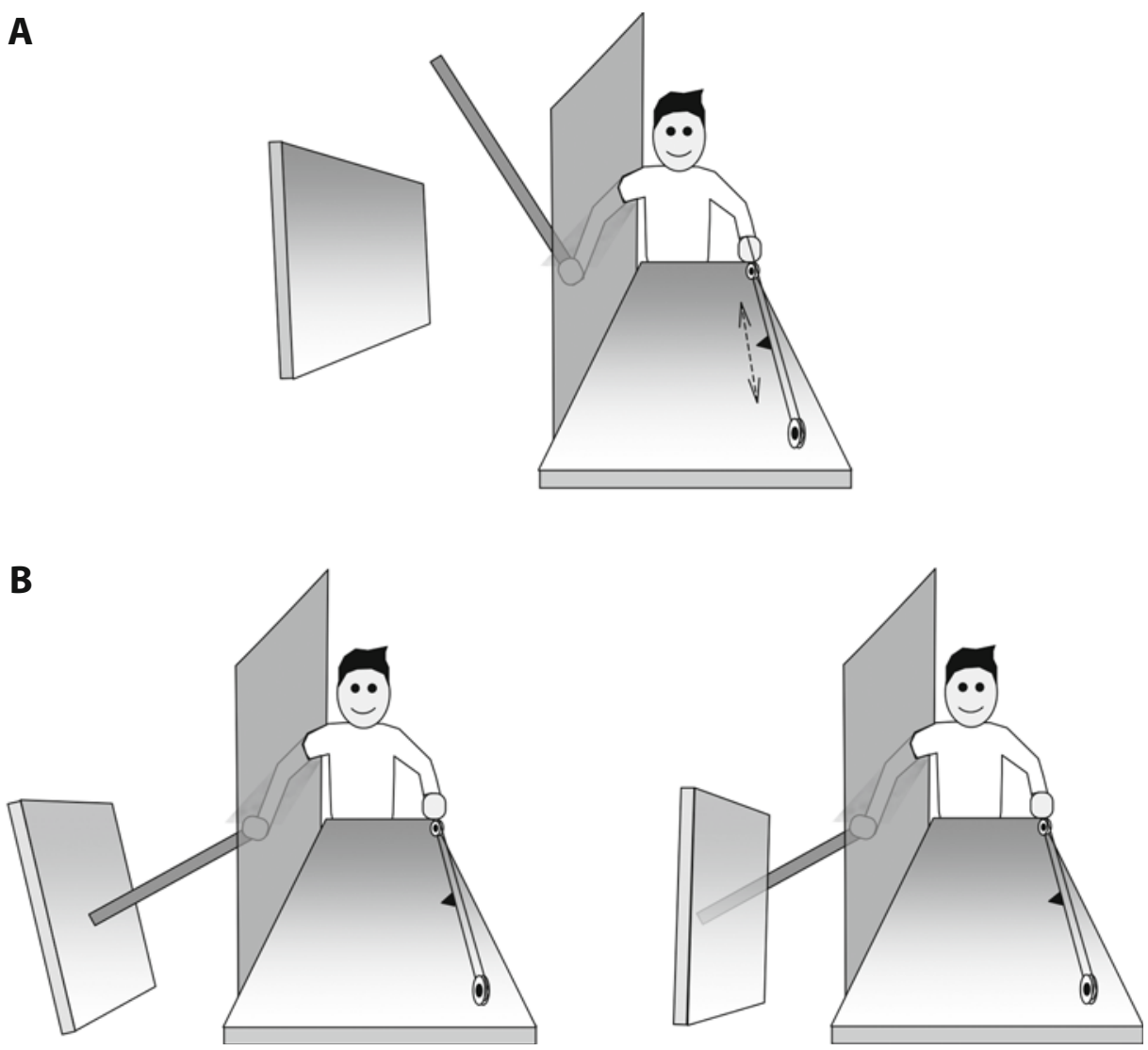

Figure 1. (A) Experimental apparatus. The participant sat at the end of a table next to a curtain occluding the view of both the wielded object and the surface to be struck. (B) The position of the surface to be struck was counterbalanced. The left panel depicts the case of rightward striking. The right panel depicts the case of leftward striking. 
Table 2

Experimental Design for Experiment 1

\begin{tabular}{ccccc}
\hline \multirow{2}{*}{ Condition } & \multicolumn{4}{c}{ Block } \\
\cline { 2 - 5 } Experimental & W & 2 & 3 & 4 \\
\hline \multirow{2}{*}{ Control } & J & J & W & W \\
& & S & S & J \\
& W & W & W & W \\
& J & J & J & J \\
\hline
\end{tabular}

Note-W, wielding; J, length judgment; S, striking.

Each participant was tested on four blocks of 20 trials. Each dowel was presented once per block in randomized order. Table 2 anatomizes the experimental design, listing events in each block by condition with $\mathrm{W}$ (wield) and S (strike) abbreviations. The table includes J, an abbreviation for "length judgment." The first block served as a pretest, during which the participant wielded the object and judged length but did not strike following judgment. For the control condition, all subsequent blocks were identical to the first. For the experimental condition, the second and third blocks served as striking blocks. During striking blocks, the participant was instructed to strike the dowel against an unseen surface (Figure 1A). After each judgment, the experimenter placed a piece of particleboard $(25 \mathrm{~cm}$ long $\times 25 \mathrm{~cm}$ wide $\times 2 \mathrm{~cm}$ thick) behind the curtain, secured to the back of a chair. A sheet of cork $(25 \mathrm{~cm}$ long $\times 25 \mathrm{~cm}$ wide $\times 0.5 \mathrm{~cm}$ thick) covered the particleboard to dampen sounds incident to striking and, thereby, control for any effect of acoustic information. ${ }^{1}$ Thus constructed, the surface was positioned so that its center coincided with the end of the dowel. Position of the surface with respect to the dowel was counterbalanced across participants: Half of the experimental participants were instructed to strike the surface in a rightward direction, and half of the experimental participants were instructed to strike the surface in a leftward direction (Figure 1B). The fourth block served as a posttest, identical in procedure to the pretest; experimental participants no longer struck the unseen surface following length judgments. The entire procedure lasted about $1 \mathrm{~h}$.

\section{Data Analysis}

We analyzed performance using growth curve modeling (GCM). GCM is a multiple regression (MR) technique that, like more common MR techniques, assigns $B$ coefficients to predictors to model an outcome variable. Whereas fitting independent regressions on by-trial predictors within individual blocks or conditions amplifies statistical error and does not control for any temporal dependence across blocks, collapsing across trials to model by-block predictors ignores any effect of by-trial predictors. There is no need, with GCM, to sacrifice unbiased estimation of either by-block or by-trial predictors. GCM allows a hierarchical (i.e., multilevel) structure and simultaneous testing of both by-block and by-trial predictors. By-block and by-trial predictors coexist in one model, minimizing statistical error and controlling for temporal dependencies at each level. This approach permits reliable estimation of the effect of time-varying predictors on performance over time (Singer \& Willett, 2003).

GCM uses maximum likelihood (ML) estimation. Ordinary leastsquares (OLS) regression techniques such as the repeated measures ANOVA assume independence of measurement and homoscedasticity. The robustness of OLS methods to heteroscedasticity guarantees only that they will compute statistics as if error were, in reality, homogeneously distributed between participants, across time, and across measurement, but there is no guarantee against distortion of the actual longitudinal structure (Molenaar, 2008). The individual differences in perceptual learning (Withagen \& van Wermeskerken, 2009) suggest heteroscedasticity in the trajectories of perceptual learning. ML estimation controls for heteroscedasticity by estimating random effects for each participant and for the finest-grain by- trial predictors. ML estimation requires evaluating improvement of model fit as a reduction in -2 LL (i.e., -2 times log-likelihood) deviance, rather than as an increment in $R^{2}$. Reductions in $-2 \mathrm{LL}$ are treated as a chi-square with degrees of freedom equal to the number of added parameters.

Although unprecedented in research on dynamic touch, GCM has already been profitably applied to other perceptual learning tasks (Blau, Stephen, Carello, \& Turvey, 2009).

\section{RESULTS}

\section{Correlation of $P L$ With $L$}

Table 3 shows mean Pearson correlation coefficient $r$ of $P L$ with $L$ by block and by condition. These $r$ s do not suggest a relative advantage for experimental participants over control participants in the posttest. We stress that these are OLS statistics, not to be confused with the ML statistics below.

\section{Definition of Predictors}

To test for a reduction in discrepancy between $P L$ and $L$, we used a number of predictors: $\log \left(I_{1}\right)$, block, strike, and hadS. $\log \left(I_{1}\right)$ represents the logarithm of the first moment of inertia (i.e., the greatest resistance to rotational acceleration about an axis through the end of the object). Block represents mere ongoing practice. Strike is coded 0 or 1 for blocks in which participants did not strike or did strike, respectively, the unseen surface (i.e., strike $=0$ for the entire control condition, and strike $=1$ for the middle two blocks of the experimental condition). HadS is coded as 0 for all blocks except the fourth, in which it is coded 1 or 0 for those participants who had or had not, respectively, struck the unseen surface. Strike and hadS both carry slightly different information about the participant's experience with mechanical information from striking. Strike carries concurrent information; hadS carries past information, allowing a rigorous comparison of the experimental condition's performance relative to the control condition in the posttest. Table 4 outlines these predictors.

Table 3

Mean Correlation Coefficients of $P L$ With $L$ by Block and by Condition

\begin{tabular}{|c|c|c|c|c|c|c|c|c|}
\hline \multirow[b]{3}{*}{ Condition } & \multicolumn{8}{|c|}{ Block } \\
\hline & \multicolumn{2}{|c|}{1} & \multicolumn{2}{|c|}{2} & \multicolumn{2}{|c|}{3} & \multicolumn{2}{|c|}{4} \\
\hline & $M$ & $S E$ & $M$ & $S E$ & $M$ & $S E$ & $M$ & $S E$ \\
\hline Experimental & .90 & .03 & .93 & .01 & .94 & .01 & .94 & .01 \\
\hline Control & .92 & .01 & .92 & .01 & .94 & .01 & .95 & .01 \\
\hline
\end{tabular}

Table 4

Description of Predictors Used in the Growth Curve

\begin{tabular}{ll}
\hline \multicolumn{1}{c}{ Predictor } & \multicolumn{1}{c}{ Description } \\
\hline $\log \left(I_{1}\right)$ & Linear effect of the logarithm of $I_{1}$ \\
$\log \left(I_{1}\right) * \log \left(I_{1}\right)$ & Quadratic effect of the logarithm of $I_{1}$ \\
Block & Block number \\
Strike & If 1, participant strikes unseen surface. \\
& If 0, participant does not strike unseen surface. \\
HadS & 1 for experimental participants in Block 4 \\
& 0 for control participants in Block 4
\end{tabular}


Control, Block $=1$, Strike $=0$, HadS $=0$

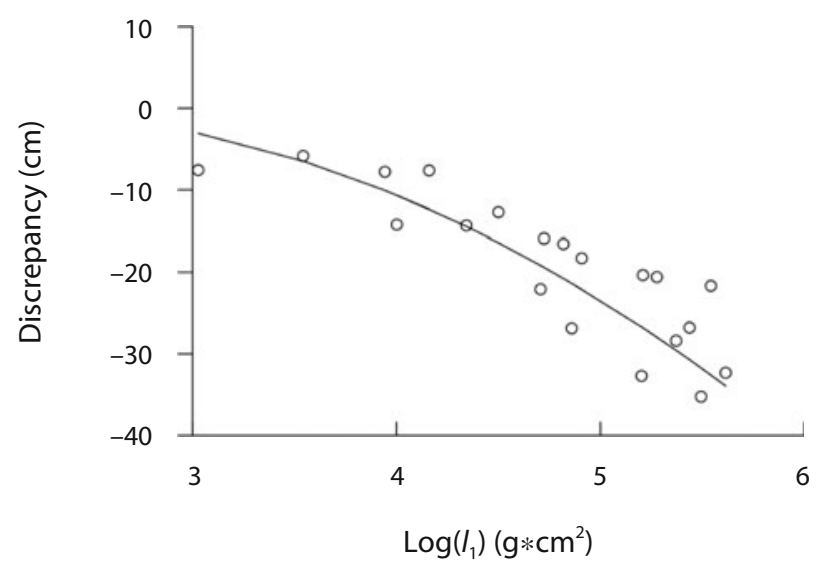

Control, Block $=3$, Strike $=0, \mathrm{HadS}=0$

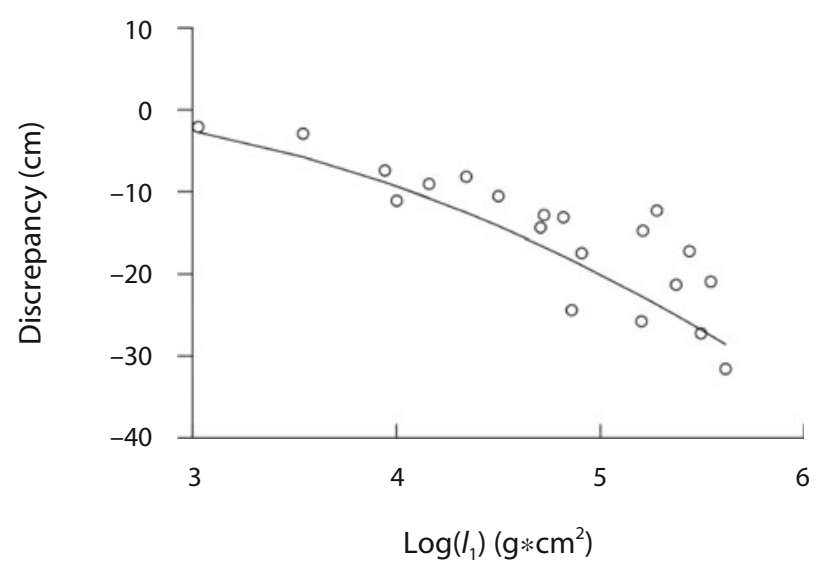

Control, Block $=2$, Strike $=0$, HadS $=0$

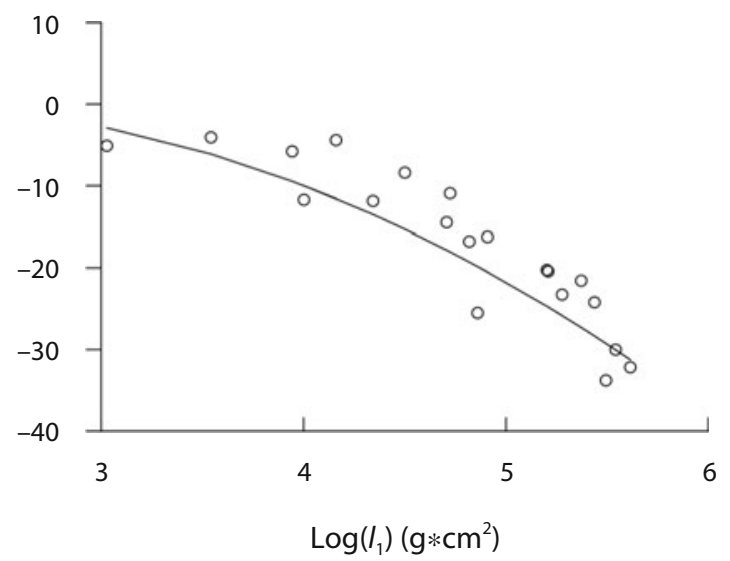

Control, Block $=4$, Strike $=0$, HadS $=0$

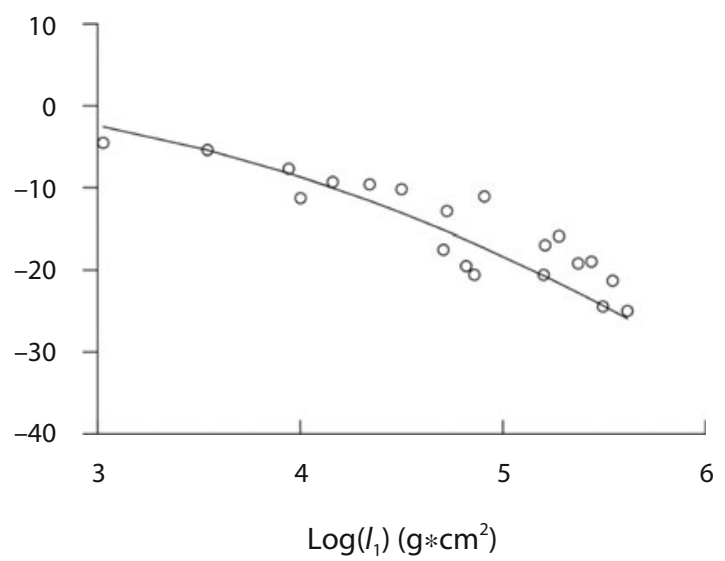

Figure 2. Plots of mean discrepancy by the logarithm of the first moment of inertia $\left(I_{1}\right)$ for the control condition. Circles represent mean discrepancy. The curve represents the predictions from the growth curve reported in the text (see Table 5).

Because the dowels are homogeneous in width, we will neither model change in variable use nor consult the variables $I_{3}$ (i.e., the third principal moment of inertia) and $P$ (length to center of percussion), for reasons of their collinearity with $I_{1}(r=1)$ and equivocality, respectively (Carello et al., 1992).

\section{Building the Growth Curve}

Figures 2 and 3 show mean discrepancy ( $P L$ minus $L$ ) for each available value of $\log \left(I_{1}\right)$ in each block for the control and experimental conditions, respectively. All mean discrepancies were, by and large, negative. Underestimation is not uncommon for dynamic touch (e.g., Withagen $\&$ Michaels, 2005). It appeared that a moderately negative quadratic relationship between $\log \left(I_{1}\right)$ and discrepancies of $P L$ from $L$ held throughout the experiment for the control condition (see Figure 2). On the other hand, the linear relationship between $\log \left(I_{1}\right)$ gave way, in the experimental condition, to a positive quadratic relationship. As can be seen in Figure 3, the distribution of discrepancies curls upward toward the horizontal axis (i.e., zero discrepancy) for experimental participants across blocks.
To quantify this change in discrepancies, we used a growth curve to model, in the first place, a quadratic function of $\log \left(I_{1}\right)$ [i.e., a function of $\log \left(I_{1}\right) * \log \left(I_{1}\right)$; see Table 4] and, in addition, the effects of block, strike, and hadS as they interact with this quadratic relationship. Hence, we used GCM to test three sets of interactions: $\log \left(I_{1}\right) * \log \left(I_{1}\right) *$ block, $\log \left(I_{1}\right) * \log \left(I_{1}\right) *$ strike, and $\log \left(I_{1}\right) * \log \left(I_{1}\right) *$ hadS. All lower order terms composing these interactions were included in the model. Random effects were fit by participant and by the by-trial predictor of $\log \left(I_{1}\right)$. We again refer the reader to Table 4 for clarifications of predictor descriptions.

\section{Interpreting the Growth Curve}

The intercept $(B=13.09, S E=25.95, p=.61)$ indicates that the overall mean difference between discrepancy and model is not significant. Independent of interactions with block, strike, and hadS, there was a negative quadratic relationship between $\log \left(I_{1}\right)$ and discrepancy $(B=-2.87$, $S E=1.26, p<.05)$ for all the participants taken together.

In order to test the effect of striking, we need to capture as much of the structure in baseline behavior as we 


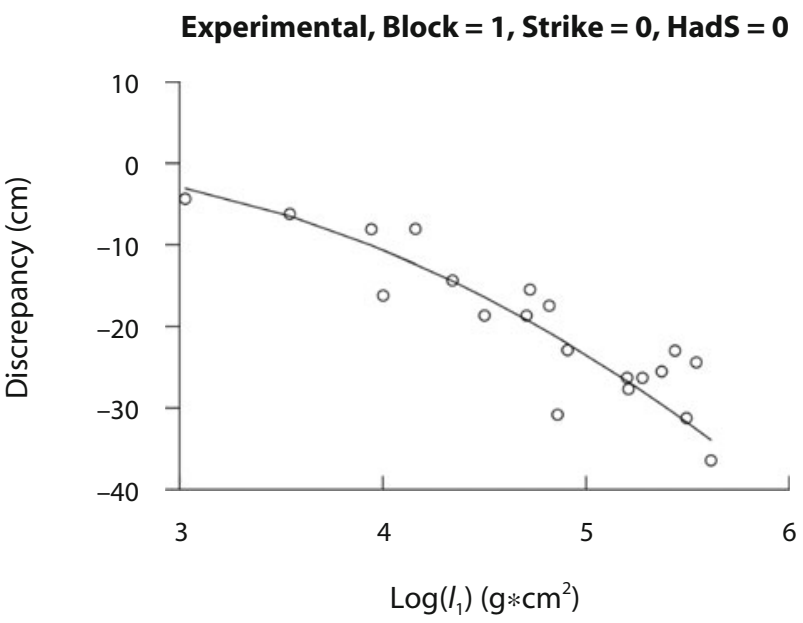

Experimental, Block $=3$, Strike $=1$, HadS $=0$

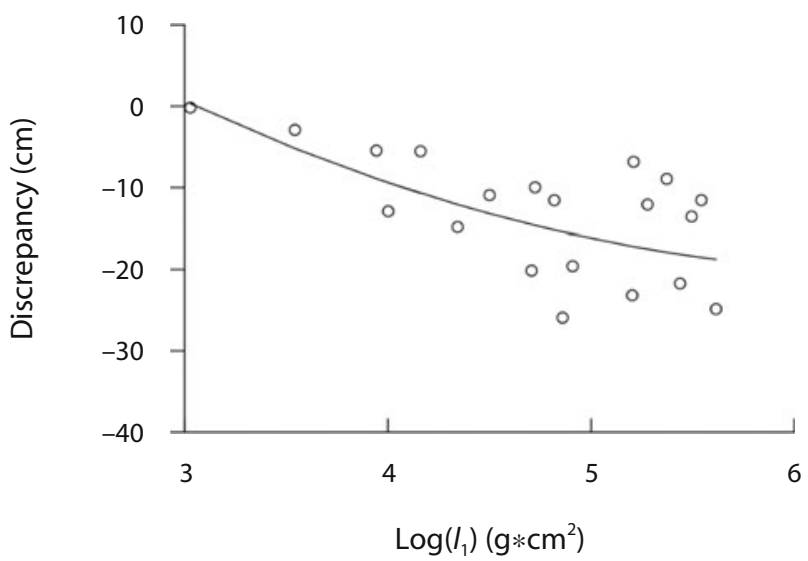

Experimental, Block $=2$, Strike $=1, \mathrm{HadS}=0$

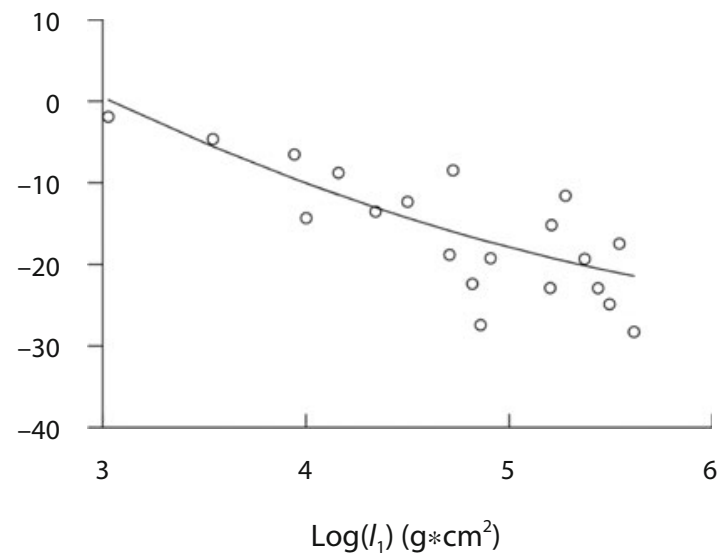

Experimental, Block $=4$, Strike $=0, \mathrm{HadS}=1$

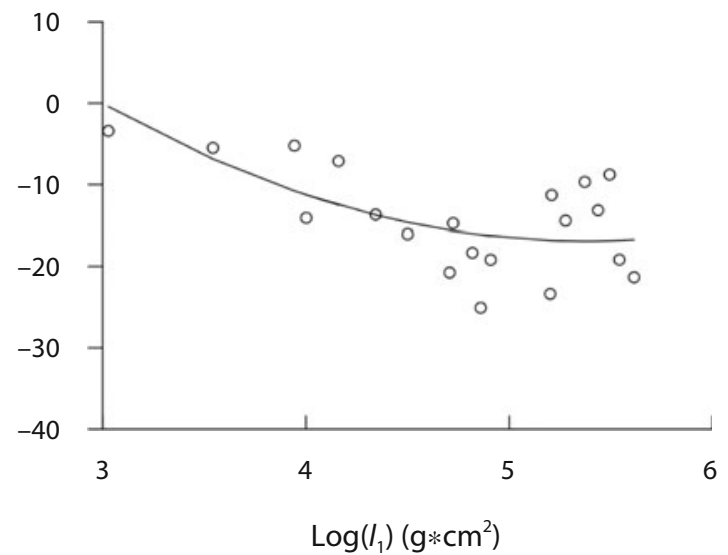

Figure 3. Plots of mean discrepancy by the logarithm of the first moment of inertia $\left(I_{1}\right)$ for the experimental condition. Circles represent mean discrepancy. The curve represents the predictions from the growth curve reported in the text (see Table 5).

can before testing for an effect of striking on behavior. A negative linear term for $\log \left(I_{1}\right)$ significantly predicts the relationship between discrepancy and $\log \left(I_{1}\right)$. The relative improvement of model fit on inclusion of the quadratic term is no indication that the quadratic of $\log \left(I_{1}\right)$ is a different, privileged informational variable. Because lower order terms precede higher order terms in a regression model, the linear term is the primary foundation for the model. The relationship suggests only that discrepancy before training increased with length, a finding with precedent (e.g., Figure 3 in Withagen \& Michaels, 2004). The quadratic function served as a baseline from which to test effects of experimental manipulations.

There were no effects of block, indicating that there was no change in discrepancy due purely to continued practice. The positive main effect of strike $(B=56.55, S E=23.18$, $p<.05$ ) indicates an overall shift of (negative) discrepancy toward zero. The significant interactions of strike with the linear $(B=-28.60, S E=10.24, p<.01)$ and the quadratic $(B=3.61, S E=1.12, p<.05) \log \left(I_{1}\right)$ terms indicate that the quadratic relationship between discrepancy and $\log \left(I_{1}\right)$ becomes more positive when participants have the benefit of mechanical information from striking. It is important to notice that the more positive quadratic relationship indicates a reduction of discrepancy between $P L$ and $L$ for the longer dowels. In the posttest, hadS makes an independent test of the quadratic relationship between the control and experimental conditions. As for strike, there was a positive main effect of hadS $(B=73.83, S E=35.42$, $p<.05)$, pushing discrepancy toward zero. The significant interactions of hadS with the linear $(B=-37.90, S E=$ $15.64, p<.05)$ and the quadratic $(B=4.70, S E=1.71$, $p<.01)$ indicate a strengthening of the positive quadratic relationship between discrepancy and $\log \left(I_{1}\right)$. That is, the effect of having had mechanical information from striking an unseen surface extends into the posttest. ${ }^{2}$

To illustrate these models, we show model predictions as solid curves in Figures 2 and 3. These curves are the addition of the $B$ coefficients (in Table 5) multiplied by the corresponding predictor variables for each stimulus presentation. The curves represent aggregate betweengroups differences in a single model that encompassed both by-block and by-trial predictors. Departures of the individual means from the predicted curve simply reflect 
Table 5

Coefficients in Growth Curve Model of the Relationship Between Discrepancy and $\log \left(I_{1}\right)$

\begin{tabular}{lrrrrr}
\hline \multicolumn{1}{c}{ Effect } & \multicolumn{1}{c}{$\Delta$} & \multicolumn{1}{c}{$S E$} & \multicolumn{1}{c}{$t$} & \multicolumn{1}{c}{$\chi^{2}(1)$} & $p$ \\
\hline Intercept & -13.09 & 25.95 & -0.50 & & .61 \\
$\log \left(I_{1}\right)$ & 11.95 & 11.46 & 1.04 & 1.10 & .30 \\
$\log \left(I_{1}\right) * \log \left(I_{1}\right)$ & -2.87 & 1.26 & -2.29 & 5.28 & $<.05$ \\
B $\operatorname{lock}$ & 2.30 & 10.44 & 0.22 & 0.05 & .83 \\
$\log \left(I_{1}\right) * \operatorname{block}$ & -1.60 & 4.61 & -0.35 & 0.12 & .73 \\
$\log \left(I_{1}\right) * \log \left(I_{1}\right) *$ block & 0.30 & 0.50 & 0.59 & 0.35 & .56 \\
Strike & 56.55 & 23.18 & 2.44 & 5.99 & $<.05$ \\
$\log \left(I_{1}\right) * \operatorname{strike}$ & -28.60 & 10.24 & -2.79 & 7.85 & $<.01$ \\
$\log \left(I_{1}\right) * \log \left(I_{1}\right) *$ strike & 3.61 & 1.12 & 3.22 & 10.41 & $<.01$ \\
$\operatorname{HadS}$ & 73.83 & 35.42 & 2.08 & 4.37 & $<.05$ \\
$\log \left(I_{1}\right) * \operatorname{hadS}$ & -37.90 & 15.64 & -2.42 & 5.91 & $<.05$ \\
$\log \left(I_{1}\right) * \log \left(I_{1}\right) *$ hadS & 4.70 & 1.71 & 2.74 & 7.57 & $<.01$ \\
\hline
\end{tabular}

random variability due to individual differences, recoverable from the participant-level random effects (Singer \& Willett, 2003) but not central for demonstrating group differences due to striking.

\section{DISCUSSION}

We predicted that mechanical information from striking would reduce the discrepancies between $P L$ and $L$ in dynamic touch. We tested this prediction in a four-block (pretest, striking, striking, posttest) experiment. In the pretest, the distribution of discrepancies reflected a negative quadratic relationship between discrepancy and $\log \left(I_{1}\right)$. Experience with striking made this relationship progressively more positively quadratic. Striking reduced discrepancy most notably for the longer dowels, to a greater extent than for the shorter dowels. Given the greater underestimation for longer stimuli in the pretest for studies testing visual feedback (e.g., Withagen \& Michaels, 2004), it is possible that the effect of striking is comparable to the effect of visual feedback. An experimental comparison of striking and visual feedback will be necessary, however.

Haptic perception can improve its own performance when multiple sources of information are available. Whereas wielding informs via mass and inertial moments, striking may inform through the elasticity of the stimuli (e.g., Kinsella-Shaw \& Turvey, 1992). For instance, the stimuli were rigid but not sufficiently dense as to damp out mechanical vibrations due to striking, and perhaps the shockwaves that propagate from the endpoint struck to the hand holding the other endpoint. Wielding and striking together support the self-training of dynamic touch. A single modality is not constrained to pick up a single kind of information (van de Langenberg, Kingma, \& Beek, 2006); it may sooner learn from the experience of multiple kinds of information within the single modality. The concept of multiple sources of information available to a single modality is not new (e.g., Todd \& Norman, 2003). However, we present evidence that these multiple sources of information may provide a basis for perceptual learning within a single modality and without the support of another. Furthermore, we provide an example of a perceptual system's generating alternative information completely by virtue of its own experience in the task. Whether from another modality or from an experimenter, explicit feedback is not necessary for perceptual learning (e.g., Karni \& Bertini, 1997). Indeed, we do not deny that striking might serve as implicit feedback, incident to the exploratory action of the perceptual system. However, such feedback remains in the sensory "language" of haptics, requires no translation from another modality, and, instead, bears an immediacy not to be found in explicit feedback.

\section{Future Directions}

Future directions for this research approach may be two-staged. The first stage should explore the range of self-training properties available in each modality. The literature on dynamic touch alone affords a wealth of phenomena that may, on closer inspection, be self-training. A comparison of standard cross-modal specification with the self-training, intramodal specification may also help define the bounds of self-training perception. The second stage should revisit cross-modal specification in selftraining terms. Gibson (1966) noted that modalities are not as separable as traditional theories of perception have suggested. The biology of a perceiving-acting organism may be differentiated but not profitably compartmentalized (Gottlieb, 1998; Van Orden et al., 2003). For example, the modality of vision is bound up in the musculoskeletal apparatus that is traditionally assigned to haptics: an upright-standing postural system to house the ocular system and a locomotory system to produce optic flow. In this light, perhaps cross-modal specification might be better understood as the self-training of a single perceptual system spanning, at least, both the visual and the haptic systems (see Streit et al., 2007).

\section{AUTHOR NOTE}

The authors extend thanks to Steven Harrison for generating the research and enthusiasm that inspired this project and to Claire Michaels for her insight, criticism, and patience during many invaluable hours of lively debate. The authors also thank Michael Turvey and three anonymous reviewers for their helpful comments on an earlier version of the manuscript. Correspondence concerning this article should be addressed to D. G. Stephen, Department of Psychology, University of Connecticut, 406 Babbidge Road, Unit 1020, Storrs, CT 06269-1020 (e-mail: damian .stephen@uconn.edu).

\section{REFERENCES}

BAHRICK, L. E., \& LiCKLITER, R. (2002). Intersensory redundancy guides early perceptual and cognitive development. In R. Kail (Ed.), Advances in child development and behavior (Vol. 30, pp. 153-187). New York: Academic Press.

Barac-Cikoja, D., \& Turvey, M. T. (1991). Perceiving aperture size by striking. Journal of Experimental Psychology: Human Perception \& Performance, 17, 330-346.

Blau, J. J. C., Stephen, D. G., Carello, C., \& Turvey, M. T. (2009). Prism adaptation of underhand throwing: Rotational inertia and the primary and latent aftereffect. Neuroscience Letters, 456, 54-58.

Carello, C., FitzPatrick, P., \& Turvey, M. T. (1992). Haptic probing: Perceiving the length of a probe and the distance of a surface probed. Perception \& Psychophysics, 51, 580-598.

Chan, T.-C., \& Turvey, M. T. (1991). Perceiving vertical distances of surfaces by means of a hand-held probe. Journal of Experimental Psychology: Human Perception \& Performance, 17, 347-358.

Fitzpatrick, P., Carello, C., \& Turvey, M. T. (1994). Eigenvalues of 
the inertia tensor and exteroception by the "muscular sense." Neuroscience, $\mathbf{6 0}, 551-568$

GiBSON, J. J. (1966). The senses considered as perceptual systems. Boston: Houghton Mifflin.

GotTLIEB, G. (1998). Normally occurring environmental and behavioral influences on gene activity: From central dogma to probabilistic epigenesis. Psychological Review, 105, 792-802.

JACOBS, D. M., \& MichaELS, C. F. (2002). On the apparent paradox of learning and realism. Ecological Psychology, 14, 127-139.

Jacobs, D. M., \& Michaels, C. F. (2007). Direct learning. Ecological Psychology, 19, 321-349.

KARNI, A., \& BERTINI, G. (1997). Learning perceptual skills: Behavioral probes into adult cortical plasticity. Current Opinion in Neurobiology, $7,530-535$.

Kinsella-Shaw, J. M., \& Turvey, M. T. (1992). Haptic perception of object distance in a single-strand vibratory Web. Perception \& Psychophysics, 52, 625-638.

Lickliter, R., BAHRICK, L. E., \& MARKHAM, R. G. (2006). Intersensory redundancy educates selective attention in bobwhite quail embryos. Developmental Science, 9, 605-616.

Michaels, C. F., Arzamarski, R., Isenhower, R. W., \& Jacobs, D. M. (2008). Direct learning in dynamic touch. Journal of Experimental Psychology: Human Perception \& Performance, 34, 944-957.

MolenaAR, P. C. M. (2008). On the implications of the classical ergodic theorems: Analysis of developmental processes has to focus on intraindividual variation. Developmental Psychobiology, 50, 60-69.

Singer, J. D., \& Willett, J. B. (2003). Applied longitudinal data analysis: Modeling change and event occurrence. New York: Oxford University Press

Stoffregen, T. A., \& Bardy, B. G. (2001). On specification and the senses. Behavioral \& Brain Sciences, 24, 195-261.

Streit, M., Shockley, K., Morris, A. W., \& Riley, M. A. (2007). Rotational kinematics influence multimodal perception of heaviness. Psychonomic Bulletin \& Review, 14, 363-367.

TodD, J. T., \& Norman, J. F. (2003). The visual perception of 3-D shape from multiple cues: Are observers capable of perceiving metric structure? Perception \& Psychophysics, 65, 31-47.

van de Langenberg, R., Kingma, I., \& Beek, P. J. (2006). Mechani- cal invariants are implicated in dynamic touch as a function of their salience in the stimulus flow. Journal of Experimental Psychology: Human Perception \& Performance, 32, 1093-1106.

Van Orden, G. C., Holden, J. G., \& Turvey, M. T. (2003). Selforganization of cognitive performance. Journal of Experimental Psychology: General, 132, 331-351.

Wagman, J. B., Shockley, K., Riley, M. A., \& Turvey, M. T. (2001). Attunement, calibration, and exploration in fast haptic perceptual learning. Journal of Motor Behavior, 33, 323-327.

Withagen, R., \& Michaels, C. F. (2004). Transfer of calibration in length perception by dynamic touch. Perception \& Psychophysics, 66, 1282-1292.

Withagen, R., \& Michaels, C. F. (2005). The role of feedback information for calibration and attunement in perceiving length by dynamic touch. Journal of Experimental Psychology: Human Perception \& Performance, 31, 1379-1390.

Withagen, R., \& VAN WeRMESKERKEN, M. (2009). Individual differences in learning to perceive length by dynamic touch: Evidence for variation in perceptual learning capacities. Attention, Perception, \& Psychophysics, 71, 64-75.

\section{NOTES}

1. Previous research has failed to show any effect of acoustic information incident to mechanical contact in dynamic touch (Carello et al. 1992).

2. This model takes discrepancy between $P L$ and $L$ as its dependent variable and $\log \left(I_{1}\right)$ as its independent variable, but because of the additive relationship between discrepancy and $P L$ and because of the collinearity between $\log \left(I_{1}\right)$ and $L$, comparable effects of block, strike, and hadS hold even when the predictor is $L$ and when the dependent variable is $P L$. The model has been phrased so as to answer the question of whether striking an unseen surface will reduce discrepancy. The authors can make the details of those alternate models available to anyone interested.

(Manuscript received April 14, 2009; revision accepted for publication June 11,2009.) 